\title{
Predictors of Osteoporosis Screening Completion Rates in a Primary Care Practice
}

\author{
Ramona S. DeJesus, MD, Rajeev Chaudhry, MBBS, MPH, Kurt B. Angstman, MD, ${ }^{2}$ \\ Stephen S. Cha, MS, ${ }^{3}$ Sidna M. Tulledge-Scheitel, MD, MPH, Rebecca L. Kesman, MD, \\ Matthew E. Bernard, MD, ${ }^{2}$ and Robert J. Stroebel, MD
}

\begin{abstract}
The United States Preventive Services Task Force and the National Osteoporosis Foundation recommend routine osteoporosis screening for women aged 65 years or older. Previous studies have shown that the use of a clinical decision-support tool significantly improves screening rates. In a recently published study, a statistically significant improvement was found in the screening rates for eligible women with use of the tool. To evaluate whether a clinical decision-support tool independently predicts completion of osteoporosis screening tests and to identify predictors of screening completion, we examined the records of 2462 female patients who were eligible for osteoporosis screening but had no prior baseline screening and who were seen in our primary care practices in 2007 and 2008. Patient and provider characteristics and clinic visit type were identified, and their association with screening test completion was statistically analyzed using both univariate and multivariate models. Screening completion rates increased significantly from 2007 to 2008. Factors associated with increased likelihood of screening completion included race, marital status, residence, presence of comorbidity (cancer, rheumatologic disease), and the year and type of visit. Screening was less likely for women aged 80 years or older. The use of a point-of-care decision-support tool not only improved osteoporosis screening rates significantly but appeared to be an independent predictor of screening completion. It potentially can facilitate the systematic and effective delivery of preventive health services to patients in the primary care setting. (Population Health Management 2011;14:243-247)
\end{abstract}

\section{Introduction}

$\mathrm{O}$ STEOPOROSIS IS A COMMON CONDITION that affects about 8 million women in the United States. Characterized by low bone mass and structural deterioration of bone tissue, it causes increased bone fragility and poses an increased fracture risk for people older than 50 years of age. If left untreated, its debilitating consequences constitute a substantial national economic burden. ${ }^{1,2}$ To address this growing threat, the 2002 United States Preventive Services Task Force and the National Osteoporosis Foundation recommended routine screening for osteoporosis for all women aged 65 years or older. ${ }^{1,3}$ Bone mineral density measurement by dual-energy X-ray absorptiometry (DEXA) is the preferred screening test and the best predictor of fracture. ${ }^{1,2}$ The task of screening eligible patients for osteoporosis rests primarily with primary care providers.

However, despite these recommendations, the osteoporosis screening rate remains low, at $12 \%$ to $56 \%{ }^{4,5}$ The use of a clinical decision-support system has been shown to improve the screening rate for osteoporosis. ${ }^{6}$ A recently published study reviewed records of female patients who were eligible for osteoporosis screening in a primary care practice, before and after implementation of a point-of-care clinical decision-support system; it found a statistically significant improvement in screening rates with use of the tool. ${ }^{7}$ The study, however, did not look into factors that predict screening completion during clinic visits. A previously conducted retrospective review of test ordering for abdominal aortic aneurysm showed that screening was more likely to be ordered at a general medical examination than at an acute care visit or follow-up examination. ${ }^{8}$

Our study sought to identify patient and provider characteristics that predict completion of osteoporosis screening within 30 days of a clinic visit among eligible female patients in 4 primary care clinics during 2007 and 2008. The association of clinic visit type with screening test ordering also was

${ }^{1}$ Division of Primary Care Internal Medicine, Center for Innovation, and Departments of ${ }^{2}$ Family Medicine and ${ }^{3}$ Health Sciences Research, Mayo Clinic, Rochester, Minnesota. 
analyzed. We hypothesized that use of a point-of-care decision-support tool, the Generic Data Management System (GDMS), was an independent factor that would predict completion of osteoporosis screening in a primary care setting.

Mayo Clinic's Employee and Community Health practice provides primary care to more than 100,000 patients at 4 practice sites in the Rochester, Minnesota area that are staffed by 45 internists and 50 family physicians. The GDMS, a clinical decision-support tool, was adopted at all practice sites of Employee and Community Health for adults in January 2008. Details of the tool were described in a prior publication. ${ }^{7}$ The GDMS includes a rule-based application coded with national guidelines for age- and gender-specific preventive services and outcome measures for chronic diseases such as diabetes and coronary artery disease. Based on data from electronic records, GDMS provides point-of-care decision support regarding services that a patient needs at the time of the visit and in the next 90 days. It checks for prior bone density tests among women ages 65 years and older and identifies those eligible for initial screening. Under a revised workflow, a paper copy of the GDMS summary screen is included in the rooming packet for the allied health staff. Rooming personnel then place the order for DEXA for women who meet initial osteoporosis screening criteria as recommended on the GDMS. The provider activates the order after discussion with the patient.

Table 1. Univariate Analysis of Variables Associated with Test Completion, 2007 and 2008

\begin{tabular}{|c|c|c|c|c|c|c|c|c|c|c|c|}
\hline Variable & \multicolumn{2}{|c|}{$\begin{array}{c}2007 \\
\text { Screened }\end{array}$} & \multicolumn{2}{|c|}{$\begin{array}{l}2007 \text { Not } \\
\text { screened }\end{array}$} & P value & \multicolumn{2}{|c|}{$\begin{array}{c}2008 \\
\text { Screened }\end{array}$} & \multicolumn{2}{|c|}{$\begin{array}{l}2008 \text { Not } \\
\text { screened }\end{array}$} & $\mathrm{P}$ value & $\begin{array}{l}\text { Over-all } \\
\text { P value }\end{array}$ \\
\hline Age & \multicolumn{2}{|c|}{$74.2 \pm 6.62$} & \multicolumn{2}{|c|}{$77.4 \pm 8.61$} & $<.001$ & \multicolumn{2}{|c|}{$74.6 \pm 7.01$} & \multicolumn{2}{|c|}{$78 \pm 8.99$} & $<.001$ & $<.001$ \\
\hline $\begin{array}{l}\text { Severity-weighted } \\
\text { sum of diseases }\end{array}$ & \multicolumn{2}{|c|}{$2.14 \pm 2.48$} & \multicolumn{2}{|c|}{$2.01 \pm 2.37$} & 0.46 & \multicolumn{2}{|c|}{$2.08 \pm 2.57$} & \multicolumn{2}{|c|}{$2.18 \pm 2.40$} & 0.57 & 0.83 \\
\hline $\begin{array}{l}\text { Severity- and } \\
\text { age-weighted } \\
\text { sum of diseases }\end{array}$ & \multicolumn{2}{|c|}{$5.06 \pm 2.70$} & \multicolumn{2}{|c|}{$5.26 \pm 2.75$} & 0.28 & \multicolumn{2}{|c|}{$5.06 \pm 2.83$} & \multicolumn{2}{|c|}{$5.49 \pm 2.81$} & 0.035 & 0.12 \\
\hline Charlson comorbidity index & No. & $\%$ & No. & $\%$ & & No. & $\%$ & No. & $\%$ & & \\
\hline Myocardial infarction & 55 & 6 & 20 & 7 & 0.53 & 48 & 5 & 19 & 8 & 0.036 & 0.11 \\
\hline Congestive heart failure & 99 & 11 & 50 & 18 & 0.002 & 113 & 11 & 41 & 17 & 0.007 & $<.001$ \\
\hline Peripheral vascular disease & 101 & 11 & 25 & 9 & 0.29 & 108 & 10 & 25 & 10 & 0.96 & 0.74 \\
\hline Coronary vascular disease & 153 & 17 & 49 & 18 & 0.83 & 175 & 17 & 57 & 24 & 0.011 & 0.07 \\
\hline Dementia & 16 & 2 & 7 & 3 & 0.44 & 16 & 2 & 7 & 3 & 0.14 & 0.43 \\
\hline Chronic pulmonary disease & 212 & 24 & 56 & 20 & 0.23 & 249 & 24 & 47 & 20 & 0.16 & 0.33 \\
\hline Ulcer & 65 & 7 & 24 & 9 & 0.45 & 79 & 8 & 19 & 8 & 0.84 & 0.89 \\
\hline Mild liver disease & 16 & 2 & 4 & 1 & 0.7 & 24 & 2 & 2 & 1 & 0.15 & 0.43 \\
\hline Diabetes, noncomplex & 186 & 21 & 70 & 25 & 0.12 & 204 & 19 & 71 & 30 & $<.001$ & 0.003 \\
\hline Diabetes, complex & 58 & 6 & 8 & 3 & 0.023 & 67 & 6 & 13 & 5 & 0.57 & 0.13 \\
\hline Hemiplegia & 11 & 1 & 4 & 1 & 0.78 & 17 & 2 & 5 & 2 & 0.62 & 0.77 \\
\hline $\begin{array}{l}\text { Renal disease, moderate } \\
\text { or severe }\end{array}$ & 98 & 11 & 39 & 14 & 0.16 & 102 & 10 & 39 & 16 & 0.004 & 0.014 \\
\hline Severe liver disease & 6 & 1 & 0 & 0 & 0.17 & 4 & 0 & 0 & 0 & 0.34 & 0.3 \\
\hline Metastatic tumor & 38 & 4 & 9 & 3 & 0.46 & 43 & 4 & 9 & 4 & 0.8 & 0.89 \\
\hline AIDS & 0 & 0 & 0 & 0 & & 0 & 0 & 0 & 0 & & \\
\hline Rheumatologic disease & 74 & 8 & 15 & 5 & 0.12 & 86 & 8 & 13 & 5 & 0.14 & 0.2 \\
\hline Cancer & 181 & 20 & 42 & 15 & 0.06 & 215 & 21 & 27 & 11 & $<.001$ & 0.002 \\
\hline Race & No. & $\%$ & No. & $\%$ & 0.012 & No. & $\%$ & No. & $\%$ & $<.001$ & $<.001$ \\
\hline •American Indian & 1 & 0 & 0 & 0 & & 1 & 0 & 0 & 0 & & \\
\hline -Black/African American & 4 & 0 & 2 & 1 & & 4 & 0 & 3 & 1 & & \\
\hline -White & 869 & 97 & 259 & 93 & & 1016 & 97 & 217 & 90 & & \\
\hline $\begin{array}{l}\text {-Native Hawaiian/Pacific } \\
\text { Islander }\end{array}$ & 0 & 0 & 0 & 0 & & 1 & 0 & 0 & 0 & & \\
\hline -Other & 3 & 0 & 0 & 0 & & 2 & 0 & 1 & 0 & & \\
\hline -Asian & 6 & 1 & 9 & 3 & & 10 & 1 & 11 & 5 & & \\
\hline •Unknown & 12 & 1 & 8 & 3 & & 13 & 1 & 8 & 3 & & \\
\hline Marital status & & & & & $<.001$ & & & & & 0.004 & $<.001$ \\
\hline •Divorced & 65 & 7 & 24 & 9 & & 83 & 8 & 23 & 10 & & \\
\hline -Married & 529 & 59 & 118 & 42 & & 615 & 59 & 109 & 45 & & \\
\hline -Single & 34 & 4 & 9 & 3 & & 42 & 4 & 10 & 4 & & \\
\hline •Widowed & 267 & 30 & 126 & 45 & & 304 & 29 & 96 & 40 & & \\
\hline Olmsted County Resident?, No. (\%) & & & & & 0.001 & & & & & $<.001$ & $<.001$ \\
\hline$\bullet$ No & 252 & 28 & 107 & 38 & & 287 & 27 & 98 & 41 & & \\
\hline •Yes & 645 & 72 & 171 & 62 & & 759 & 72 & 141 & 59 & & \\
\hline
\end{tabular}




\section{Methods}

An independent data abstractor reviewed all records of female patients aged 65 years or older who were seen in the Family Medicine and Primary Care Internal Medicine practice sites in 2007, prior to GDMS implementation, and in 2008, 1 year into GDMS implementation. Patient characteristics, which included age, sex, race, marital status, residence, comorbidity, type of clinic visit (full vs. limited examination), and provider specialty (primary care internal medicine or family medicine) were identified.

\section{Statistical analysis}

Pearson's chi-square test was used for univariate analysis to compare screening rates between 2007 and 2008 and to identify variables that predicted test completion each year. SAS GENMOD statistical analysis was conducted to analyze if any of the variables studied independently predicted completion of osteoporosis screening 30 days after a clinic visit. All statistical analyses were performed with SAS version 9.1.3 software (SAS Institute, Inc., Cary, NC). A $P$ value of $<0.05$ was considered statistical significant.

\section{Results}

A total of 2462 women who were eligible for osteoporosis screening were seen in both years. Most were white $(96 \%)$ and resided in Olmsted County (70\%); 59\% of the women were married, and $32 \%$ were widowed; $61 \%$ were seen for a full examination, while $35 \%$ had a limited examination. Family medicine providers saw $67 \%$ of patients studied; primary care internal medicine providers saw 33\%.

In 2007, $897(76.3 \%)$ of the 1128 eligible women seen in both primary care internal medicine and family medicine clinics completed the screening test for osteoporosis within 30 days of their clinic visit. The remaining 278 were not screened. A total of $1047(81.4 \%)$ of 1287 eligible patients completed screening in 2008. The difference in completion rates between the 2 years was statistically significant (2-tailed $P$ value of 0.0027 using Pearson's chi-square test).

In univariate analysis using the Charlson Comorbidity Index, age, race, marital status, residence, and the presence of 3 comorbidities (ie, congestive heart failure, uncomplicated diabetes, cancer) were statistically significant predic- tors of osteoporosis screening completion $(P$ value: $<0.005)$. The likelihood of missed screening was lower in the presence of these variables (Table 1). The severity-weighted sum of diseases was not associated with test completion; however, when age was added to the severity-weighted sum of diseases, there was a statistically significant association with test ordering, but only in $2008(P=0.035)$.

Using the multivariate model, age, race, marital status, residence, and the presence of a comorbidity (ie, cancer, rheumatologic disease) were independent predictors of screening test completion. Women who were white, married, and residents of Olmsted County were more likely to be screened $(P=<0.005)$. Likewise, women with a diagnosis of cancer or rheumatologic disease were $50 \%$ more likely to complete screening (odds ratio [OR]: 0.5). The year and type of clinic visit also significantly predicted test completion. Patients seen in 2008 for full or general examinations were more likely to be screened (Table 2). Interestingly, age was inversely correlated to test completion; screening was more likely to be missed in older persons, specifically those aged 80 years or older (OR: 1.059). The provider's specialty did not predict test completion $(P=0.16)$.

\section{Discussion}

In uinvariate analysis, age and presence of chronic illness (eg, cancer, diabetes) were significant predictors of screening test completion during both 2007 and 2008. Older women with comorbidities are probably seen more often in their primary care clinic, which increases the opportunity to capture gaps in preventive care. The actual number of clinic visits that each patient made during each year was not examined and potentially would constitute a variable in osteoporosis screening completion rates.

With multivariate analysis, age remains an independent predictor of screening completion. While it is an established risk factor for osteoporosis and would generally trigger screening, ${ }^{9}$ this study showed advancing age to be associated with a decreased likelihood of completing the screening test; women aged 80 years or older are less likely to be screened. Indeed, increasing age by itself has been identified as a barrier to effective osteoporosis care, along with the presence of dementia, lack of treatment adherence, and inadequate social support among vulnerable elderly patients. ${ }^{10}$

Table 2. Multivariate Analysis of Variables Significantly Associated with Test Completion

\begin{tabular}{|c|c|c|c|c|c|c|c|c|}
\hline & \multirow[b]{2}{*}{ Estimate } & \multirow[b]{2}{*}{$S E$} & \multicolumn{2}{|c|}{$95 \% C I$} & \multirow[b]{2}{*}{ Odds ratio } & \multicolumn{2}{|c|}{$95 \% C I$} & \multirow[b]{2}{*}{$\mathrm{P}$ value } \\
\hline & & & Lower & Upper & & Lower & upper & \\
\hline \multicolumn{9}{|l|}{ Variables } \\
\hline 2008 & 0.3388 & 0.0792 & 0.1836 & 0.4941 & 0.713 & 0.610 & 0.832 & $<.0001$ \\
\hline Age & 0.0572 & 0.0097 & 0.0382 & 0.0762 & 1.059 & 1.039 & 1.079 & $<.0001$ \\
\hline Rheumatologic disease, No. (\%) & 0.6811 & 0.2935 & 0.1058 & 1.2563 & 0.506 & 0.285 & 0.900 & 0.0203 \\
\hline Cancer, No. (\%) & 0.5529 & 0.1706 & 0.2185 & 0.8873 & 0.575 & 0.412 & 0.804 & 0.0012 \\
\hline White & 1.2627 & 0.2837 & 0.7068 & 1.8187 & 0.283 & 0.162 & 0.493 & $<.0001$ \\
\hline Married & 0.3952 & 0.1354 & 0.1298 & 0.6605 & 0.674 & 0.517 & 0.878 & 0.0035 \\
\hline Olmsted resident & 0.5901 & 0.1321 & 0.3312 & 0.849 & 0.554 & 0.428 & 0.718 & $<.0001$ \\
\hline Full examination & 0.2322 & 0.113 & 0.0107 & 0.4537 & 0.793 & 0.635 & 0.989 & 0.0399 \\
\hline
\end{tabular}

CI, confidence interval; SE, standard error. 
Likewise, it is common practice for women in this age group who present with vertebral or hip fracture to be clinically treated for osteoporosis without a screening test. Decreased life expectancy may also limit screening. One study identified greater concern about developing osteoporosis and better knowledge of bone mineral density (BMD) testing as 2 determinants of the readiness to undergo osteoporosis screening in older, high-risk patients. ${ }^{11}$ A closer look at factors that account for decreased screening among elderly patients in this study would be useful in designing effective practice interventions to improve osteoporosis diagnosis and management.

Race and residence are also significantly associated with increased screening rates. Because more than two thirds of the women in this study are white and reside in predominantly white Olmsted County, the results could have been skewed. However, the data were unchanged even in the final, stable multivariate model. There was a trend toward increased test completion among Asian women in 2008, but the number is too low to determine statistical significance.

Two diseases, cancer and rheumatologic illness, are associated with higher screening rates. Other studies have observed the correlation between osteoporosis and the presence of these conditions because of the underlying disease pathology and/or treatment (eg, corticosteroids). ${ }^{12}$ Therefore, their presence is likely to trigger an order for a DEXA test. Patients with these comorbidities also require frequent clinic visits, allowing greater opportunity to screen.

There was a statistically significant increase in the osteoporosis screening rate from 2007 to 2008. This result confirmed the finding of a previous study, which showed that use of a clinical decision-support tool significantly improved the osteoporosis screening rate among eligible women seen in the primary care setting. ${ }^{7}$

Screening of eligible women is likely to be completed after a full examination. This finding was also observed in a previous study that looked at screening orders for abdominal aortic aneurysm in a primary care practice; the test was more likely to be ordered at a general or full examination than at an acute care or follow-up examination. ${ }^{8}$ More time is usually allotted for a full examination, which provides a greater opportunity to address preventive health screening.

The characteristics and number of women seen for full and limited examinations in both years were similar; the only factor that would account for the statistically significant association of the year 2008 with screening completion was the availability of GDMS. This finding reflects the utility of the tool in improving preventive care practice across primary care population groups and supports the hypothesis that it appears to be an independent predictor of osteoporosis screening completion.

More eligible patients were seen in family medicine clinics than in primary care internal medicine; however, test ordering did not differ statistically between the two specialty areas. This also was observed in the retrospective study on abdominal aortic aneurysm screening in which no difference was seen in the ordering rates among providers with different roles or of different sexes. ${ }^{8}$

This study identified factors that predict osteoporosis screening completion by primary care providers in an academic institution in the Midwestern United States, and results may not be generalizable to community-based primary care practices in other geographic areas. Most of the women in the study were white, which limits the application of results to other ethnic and minority groups. It would be interesting to see results from a larger, multicenter study with a more diverse ethnic population.

\section{Conclusions}

Independent predictors of increased likelihood of osteoporosis screening completion among women aged 65 years or older include ethnicity (white), marital status (married), and the presence of certain comorbidities (ie, cancer, rheumatologic disease). There is a decreased likelihood of screening completion with advancing age. Screening is more likely to be done during full examination visits and with use of a point-of-care clinical decision-support tool. Use of such a tool significantly improved osteoporosis screening rates for an eligible patient group seen in a primary care practice and appears to independently predict screening completion.

\section{Acknowledgments}

The authors acknowledge the Mayo Foundation for funding this study and manuscript preparation.

\section{Author Disclosure Statement}

Dr. Chaudhry is an employee of Mayo Clinic and the inventor of the GDMS software referenced in this publication. Mayo Clinic has licensed this technology to a commercial entity (VitalHealth Software) but to date has received no royalties. Dr. Chaudhry receives no royalties from the licensing of this technology. Drs. DeJesus, Angstman, Tulledge-Scheitel, Kesman, Bernard, and Stroebel, and $\mathrm{Mr}$ Cha disclosed no conflicts of interest.

\section{References}

1. National Osteoporosis Foundation. Clinician's Guide to Prevention and Treatment of Osteoporosis. Washington, DC: National Osteoporosis Foundation; 2008.

2. US Department of Health and Human Services. Bone Health and Osteoporosis: A Report of the Surgeon General. Rockville, MD: US Dept of Health and Human Services, Public Health Service, Office of the Surgeon General; 2004.

3. US Preventive Services Task Force (USPSTF). Screening for osteoporosis in postmenopausal women: Recommendations and rationale. Ann Intern Med. 2002;137:526-528.

4. Gallagher TC, Geling O, Comite F. Missed opportunities for prevention of osteoporotic fracture. Arch Intern Med. 2002;162:450-456.

5. Cohen K, Maier D. Osteoporosis: evaluation of screening pattern in a primary-care group practice. J Clin Densitom. 2008;11:498-502.

6. Kastner M, Straus SE. Clinical decision support tools for osteoporosis disease management: A systematic review of randomized controlled trials. J Gen Intern Med. 2008;23: 2095-2105.

7. DeJesus RS, Angstman KB, Kesman R, et al. Use of a clinical decision support system to improve osteoporosis screening. J Eval Clin Pract. Epub ahead of print August 10, 2010. doi: 10.1111/j. 1365-2573.2010.01528.x.

8. Eaton J, Reed D, Angstman K, et al. Effect of visit length and a clinical decision support tool on abdominal aortic screening rates in a primary care practice: A retrospective review. J Eval Clin Pract. In press. 
9. Barrett-Connor E, Weiss TW, McHorney CA, Miller PD, Siris ES. Predictors of falls among postmenopausal women: Results from the National Osteoporosis Risk Assessment (NORA). Osteoporosis Int. 2009;20:715-722.

10. Switzer JA, Jaglal S, Bogoch ER. Overcoming barriers to osteoporosis care in vulnerable elderly patients with hip fractures. J Orthop Trauma. 2009;23:454-459.

11. Polinski JM, Cadarette SM, Arnold M, et al. High-risk patients' readiness to undergo BMD testing for osteoporosis diagnosis in Pennsylvania. Int Q Community Health Educ. 2008-2009;29:223-240.

12. Sinigaglia L, Nervetti A, Mela $Q$, et al. A multicenter cross sectional study on bone mineral density in rheumatoid ar- thritis: Italian Study Group on Bone Mass in Rheumatoid Arthritis. J Rheumatol 2000;27:2582-2589.

Address correspondence to: Ramona S. DeJesus, M.D. Division of Primary Care Internal Medicine Mayo Clinic 200 First St. S.W. Rochester, MN 55905

E-mail: dejesus.ramona@mayo.edu 
